\title{
Estabilidad del Carotenoide Licopeno en Tomates en Conserva
}

\author{
Alicia L. Ordóñez, María E. Balanza, Fanny R. Martín y Cecilia A. Flores \\ Universidad Nacional de Cuyo, Facultad de Ciencias Aplicadas a la Industria, Bdo. de Irigoyen 357, \\ San Rafael, Mendoza-Argentina (e-mail: aordonez@fcai.uncu.edu.ar)
}

\begin{abstract}
Resumen
El objetivo de este trabajo fue determinar la estabilidad del carotenoide licopeno durante el proceso de elaboración de conservas de tomates peritas y evaluar la misma durante su almacenamiento como producto terminado. Se trabajó con muestras provenientes de elaboraciones industriales extraídas en distintos puntos del proceso: tomates frescos, en la boquilla de alimentación de la línea; tomate pelado, a la salida de la peladora termofísica y producto terminado a la salida del esterilizador-enfriador, de distintos lotes de elaboración y en tres ocasiones durante la temporada 2007. El producto terminado, envasado en hojalata, fue evaluado durante un año, cada tres meses. El licopeno fue extraído con una mezcla de hexano-acetona-etanol y determinado por espectrofotometría visible a $472 \mathrm{~nm}$. Los resultados se analizaron estadísticamente mostrando que la esterilización industrial produce liberación celular del licopeno.
\end{abstract}

Palabras clave: licopeno, tomates en conserva, estabilidad, carotenoide, almacenamiento

\section{Lycopene Carotenoide Stability in Canned Tomatoes}

\begin{abstract}
The objective of this work was to determine lycopene carotenoid stability during manufacturing process in canned peeled whole tomatoes and during its storage as final product. Samples were taken during industrial manufacturing at different process points: fresh tomatoes when they were feeding to process line, peeled tomatoes from thermophysical peeler and finished product after it passed the cooker-cooler. Samples were obtained from different manufacturing lots at three times during the 2007 harvesting season. Canned tomatoes were analyzed every three months, during one year. Lycopene was extracted with hexane-acetone-ethyl alcohol and measured by spectrophotometry at $472 \mathrm{~nm}$. Statistical analysis of the results shows that industrial sterilization produces cell release of lycopene.
\end{abstract}

Keywords: lycopene, canned tomatoes, stability, carotenoid, storage 


\section{INTRODUCCIÓN}

El licopeno, carotenoide característico del tomate, que le confiere su coloración roja, no tiene actividad provitamínica $A$, pero muestra una capacidad antioxidante dos veces mas alta que el $\beta$ caroteno, por lo que su presencia en la dieta se considera de gran interés (Olmedilla, 1999; Shi y Le Maguer, 2000) ya que actúa protegiendo las células del estrés oxidativo producido por la acción de los radicales libres que son responsables del cáncer, enfermedades cardiovasculares y envejecimiento (Candelas et al., 2005). El licopeno es un agente anticarcinogénico y antiaterogénico al intervenir en la comunicación intercelular (Fornelli et al., 2007) y modular los mecanismos inmunológicos (Lee et al., 2000; Rao, 2006).

Es un carotenoide altamente lipofílico que se caracteriza por carecer de anillos cíclicos y poseer once dobles enlaces conjugados, de estructura sencilla con una cadena alifática formada por cuarenta átomos de carbono (Nguyen y Schwartz, 1999). Su obtención por síntesis química aún no está totalmente establecida y, a diferencia de otros carotenoides como el $\beta$-caroteno producido a gran escala por síntesis, el licopeno se obtiene fundamentalmente a partir de fuentes naturales, hongos y muy especialmente tomates. Sin embargo, los sistemas de extracción son costosos y el licopeno presenta una baja estabilidad, lo que ha limitado su utilización como colorante alimenticio. Se absorbe mejor a través de las grasas y aceites por su liposolubilidad y se encuentra presente en el organismo humano tanto en sangre como en tejidos (Perking-Veazie et al., 2001).

En España se realizó un estudio de fuentes de vitamina C, E y carotenoides específicos y establecieron que los tomates son los primeros como fuentes de licopeno (Raffo et al., 2006). Nguyen y Schwartz (1999), mencionan que en el tomate maduro, el carotenoide mayoritario es el licopeno (aproximadamente $83 \%$ ) y en porcentaje también importante, se encuentra el ß-caroteno (de 3 a $7 \%$ ). Shi y Le Maguer (2000) han demostrado que el contenido de licopeno aumenta con la maduración de los tomates y puede presentar grandes diferencias según la variedad, condiciones del cultivo como el tipo de suelo y clima, tipo de almacenamiento, etc. Estos autores reportan concentraciones de licopeno en tomates de ensalada alrededor de $30 \mathrm{mg} / \mathrm{Kg}$ y en los de "tipo pera" más de diez veces esa cifra. Estudios realizados en la provincia de Entre Ríos, Argentina, informaron concentraciones promedio de licopeno en tomate redondo en las fases de maduración y comercialización de 39,05 \pm 4,41 mg/Kg y 49,19 \pm 6,90 mg/Kg respectivamente (Zapata et al., 2007).

El licopeno, así como muchos otros carotenoides naturales, existe en las formas isómeras all-trans y cis. En tomate fresco al estado natural el $90 \%$ corresponde a isómeros trans (Lambelet et al., 2008) mientras que los productos procesados del tomate contienen mayor proporción de isómeros cis (Schierle et al., 1997). El tratamiento térmico es responsable por esta isomerización inducida (Van het Hof et al., 2000).

Desde el punto de vista nutricional el problema más grande que tiene la ingesta de licopeno está en cómo consumirlo. Su biodisponibilidad es mejor cuando se somete a cocción (pasta de tomate) mejorando sus propiedades antioxidantes y anticancerígenas (Méndez y Hernández, 2006). El tomate procesado presenta mejor absorción (cerca de cuatro veces más) que los tomates frescos (Cohn et al., 2000), de ahí la importancia de su consumo como conserva. Diversos factores físicos y químicos afectan la estabilidad de los carotenoides, especialmente temperaturas elevadas, exposición a la luz, al oxígeno y a pH extremos (Nguyen y Schwartz, 1999; Shi y Le Maguer, 2000).

Uno de los factores que más afecta la biodisponibilidad de los carotenoides es su liberación de la matriz física (alimento) en la cual son ingeridos y su disolución en la fase lipídica (Parker, 1996). El tratamiento térmico de hortalizas y verduras, parece mejorar la biodisponibilidad de los carotenoides en muchos alimentos (Micozzi et al., 1992; Granado et al., 1992; Stahl y Sies, 1992; RodríguezAmaya, 1997; Gartner et al., 1997), probablemente al disociar los complejos proteicos y promover la rotura de paredes celulares inducida por tratamiento térmico.

El proceso de elaboración de tomates en conserva es una apertización y consta de las siguientes etapas: recepción de materia prima, inspección, lavado, pelado (escaldado y pellizcado), inspección, envasado, dosificación de líquido de gobierno (jugo de tomate) con pre-vacío, remachado, 
esterilización, enfriamiento, etiquetado y almacenamiento. Es durante este proceso que el contenido de licopeno puede sufrir cambios en su concentración y/o biodisponibilidad.

A la fecha no se han encontrado publicaciones científicas que muestren los cambios que sufre el licopeno, durante el proceso de elaboración de conservas de tomate perita, en el que la materia prima sufre una fuerte exposición a distintos factores físicos y químicos, principalmente a la acción del calor, antes de trasformarse en producto terminado.

El objetivo de este trabajo es determinar la estabilidad del carotenoide licopeno durante el proceso de elaboración de conservas de tomates peritas pelados enteros y evaluar la misma durante su almacenamiento como producto terminado.

\section{MATERIALES Y MÉTODOS}

Para determinar la estabilidad del licopeno durante el proceso de elaboración de tomates en conserva se trabajó con muestras elaboradas a nivel industrial en fábrica de conservas La Colina S.A., San Rafael, Mendoza durante los meses de febrero y marzo del 2007.

Se seleccionaron para el estudio tres puntos a lo largo del proceso de elaboración, los que fueron considerados críticos en relación a la estabilidad del licopeno. Se extrajeron muestras de: (1) tomates frescos en la boquilla de alimentación de la línea, (2) tomates pelados a la salida de la peladora termofísica (temperatura de escaldado: $97^{\circ} \mathrm{C}, 40$ segundos) y (3) producto terminado (envase de hojalata) a la salida del esterilizador-enfriador, Cooker-Cooler (temperatura: $98^{\circ} \mathrm{C}, 58$ minutos). Las muestras fueron extraídas por duplicado y trasladadas inmediatamente al laboratorio de investigación de la Facultad de Ciencias Aplicadas a la Industria para su análisis. Las muestras del punto dos fueron trasladadas en envases de hojalata con tapas de papel de aluminio para evitar interferencias en el estudio por acción de la luz. Esta experiencia se repitió con distintos lotes de elaboración y en tres oportunidades diferentes durante la temporada de elaboración. Las muestras de producto terminado de los distintos lotes fueron almacenadas a temperatura ambiente durante un año, evaluando el contenido de licopeno cada tres meses.

El carotenoide licopeno fue determinado por espectrofotometría visible. Para su extracción se utilizo la técnica de Sadler et al. (1990). Se pesó aproximadamente 1g de muestra en un Erlenmeyer, se lo cubrió de la luz con papel aluminio, se agregaron $25 \mathrm{ml}$ de solvente (hexano: acetona: etanol; en una proporción 2:1:1) y se agitó magnéticamente durante 30 minutos. Luego se adicionaron $10 \mathrm{ml}$ de agua destilada, que separó el contenido en dos fases, una polar (agua, alcohol, acetona) y otra no polar (hexano, licopeno). A continuación se tomó la fase no polar con una pipeta Pasteur, se la colocó en una probeta (la cual se resguardó de la luz con papel aluminio) para medir el volumen extraído de hexano. Se adicionaron $25 \mathrm{ml}$ más de solvente al erlenmeyer para realizar una segunda extracción repitiendo el procedimiento descripto anteriormente. Se reunió todo el hexano extraído y se midió su volumen. A partir de allí se realizaron diluciones para la medición espectrofotométrica a una longitud de onda de $472 \mathrm{~nm}$.

Para la cuantificación se construyó una curva de calibración utilizando una muestra de reactivo patrón (DMS, redivivo TM, lycopene 10\% WS) que contiene 10\% de licopeno (soluble en agua). Se prepararon soluciones de licopeno patrón a 0,$2 ; 0,3 ; 0,5 ; 0,8 ; 1,0 ; 1,5$ y $2,0 \mathrm{mg} / \mathrm{l}$. Las mismas cumplieron con la Ley de Lambert y Beer con un alto grado de ajuste $\left(R^{2}=0.9997\right)$.

\section{RESULTADOS Y DISCUSIÓN}

La concentración promedio y desviación estandar del contenido de licopeno, de tomates peritas con piel fue de $111,5 \pm 4,37 \mathrm{mg} / \mathrm{kg}$ lo que estaría indicando que los valores obtenidos son superiores a los reportados en tomates de ensalada: $30 \mathrm{mg} / \mathrm{Kg}$ y $49,9 \mathrm{mg} / \mathrm{Kg}$ por Shi y Le Maguer (2000) y Zapata et al. (2007) respectivamente, lo que confirmaría que se pueden presentar grandes diferencias según la variedad, condiciones del cultivo como el tipo de suelo y clima, tipo de almacenamiento, etc.

En la tabla 1 se muestra el análisis estadístico de la variación del contenido de licopeno durante el proceso de elaboración de tomates en conserva, cuantificando 111,5 $\pm 4,37 \mathrm{mg} / \mathrm{kg}$ en la boquilla de 
alimentación de la línea (tomate perita con piel); 90,2 $\pm 6,65 \mathrm{mg} / \mathrm{kg}$ a la salida de la escaldadora (tomate sin piel) y $98,36 \pm 4,63 \mathrm{mg} / \mathrm{kg}$ a la salida del esterilizador enfriador coker-cooler (producto terminado). EI ANOVA y prueba de rangos múltiples de Tuckey HSD, pone de manifiesto diferencias muy significativas para un nivel de confianza de $99 \%$ ( $p$-valor $<0.01)$ en las concentraciones de licopeno entre las distintas etapas del proceso no presentando homogeneidad de grupo.

Tabla 1: Resumen estadístico de la variación del contenido de Licopeno (mg/Kg de muestra).

\begin{tabular}{|l|l|l|l|l|l|}
\hline \multirow{2}{*}{$\begin{array}{c}\text { Operaciones en el } \\
\text { proceso }\end{array}$} & \multicolumn{4}{|c|}{ Análisis estadístico } \\
\cline { 2 - 6 } & \multicolumn{1}{|c|}{$\begin{array}{c}\text { Media } \\
\text { estándar }\end{array}$} & $\begin{array}{c}\text { Desviaciónimo } \\
\text { estáno }\end{array}$ & p-valor \\
\hline 1- Tomate fresco & $111,5^{(a)}$ & 4,37 & 106 & 118 & \multirow{2}{*}{0,000} \\
\hline 2- Tomate escaldado & $90,2^{(\mathrm{b})}$ & 6,65 & 81 & 100 & \\
\hline 3- Producto terminado & $98,36^{(\mathrm{c})}$ & 4,63 & 92 & 107 & \\
\hline
\end{tabular}

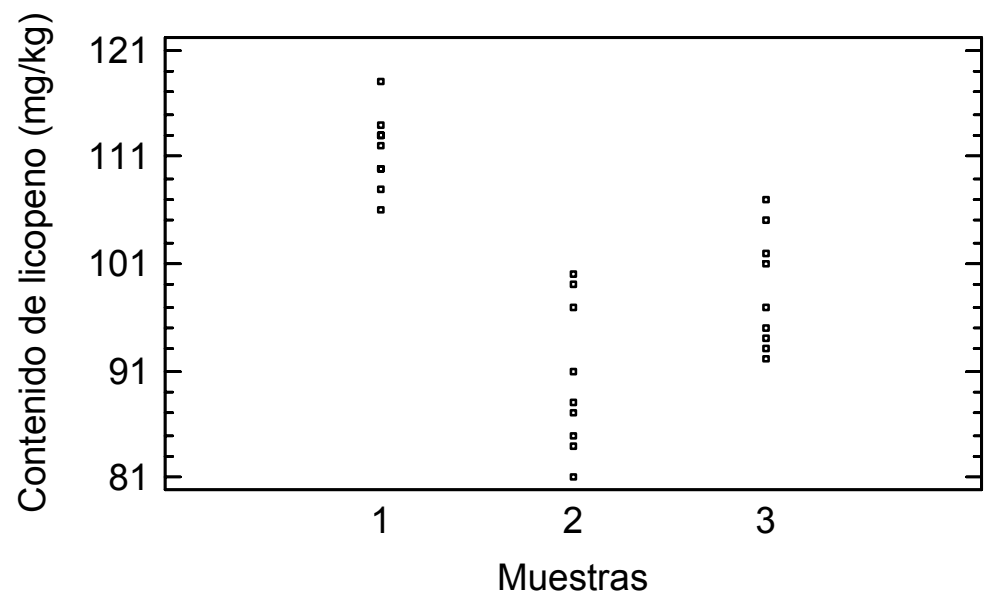

Fig. 1: Gráfico de dispersión de los contenidos de licopeno de tomate fresco, escaldado y terminado, en el proceso de elaboración.

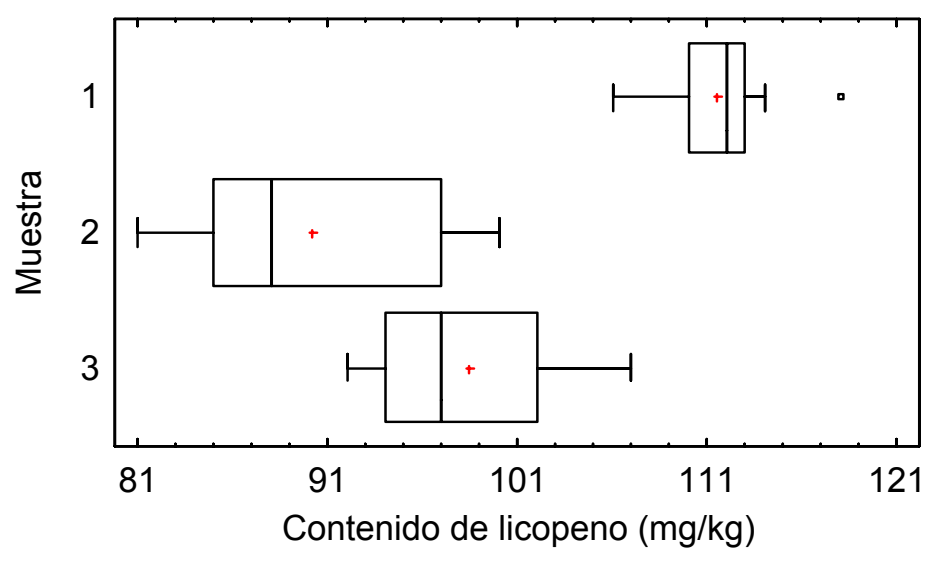

Fig. 2: Gráfico de Cajas y Bigotes de los contenidos de licopeno de tomate fresco, escaldado y terminado, en el proceso de elaboración.

La fig. 1 muestra la dispersión de los valores de la concentración de licopeno obtenidos en las diferentes etapas de elaboración.

En la fig. 2 se observa con claridad una reducción significativa del contenido de licopeno después del pelado por escaldado. Estos resultados muestran que en la epidermis de los tomates hay un alto contenido de licopeno el que se pierde en el proceso de pelado. Sin embargo, luego se observa un aumento significativo en concentración de licopeno entre tomates escaldados y el producto 
terminado, lo que estaría confirmando que el tratamiento térmico $\left(98^{\circ} \mathrm{C}, 58\right.$ minutos) actúa sobre la estructura física de la materia prima, provocando una liberación del carotenoide al promover la ruptura de paredes celulares, lo que no ocurriría en la operación de escaldado $\left(97^{\circ} \mathrm{C}, 40\right.$ segundos) por ser éste un tratamiento térmico muy corto.

Atendiendo a los resultados obtenidos y a lo reportado por Gartner et al. (1997) acerca de la mejor absorción de los principios activos del tomate procesado con respecto a tomate fresco, combinado con la mayor liberación por ruptura celular, y lo mencionado por Méndez y Hernández (2006) sobre la mejora de la biodisponibilidad, cuando se somete a cocción la pasta de tomate, se confirma que las conservas de tomate perita pelado entero son buenas proveedoras de antioxidantes.

El análisis de la evolución del contenido de licopeno (tabla 2), en muestras de tomates en conserva envasados en hojalata (al abrigo de la luz) extraídas a la salida del esterilizador - enfriador cokercooler de los lotes de elaboración en estudio y almacenadas a temperatura ambiente durante un año pone de manifiesto la no variación del parámetro bajo las condiciones indicadas. Las figuras 3 y 4 confirman estadísticamente la estabilidad del carotenoide durante su comercialización.

Tabla 2: Resumen estadístico de la evolución del contenido de licopeno a lo largo de su almacenamiento a temperatura ambiente.

\begin{tabular}{|l|l|l|l|l|l|}
\hline \multirow{2}{*}{$\begin{array}{c}\text { Tiempo de } \\
\text { almacenamiento }\end{array}$} & \multicolumn{5}{c|}{ Análisis estadístico } \\
\cline { 2 - 5 } & \multicolumn{1}{|c|}{ Media } & $\begin{array}{c}\text { Desviación } \\
\text { estandar }\end{array}$ & Mínimo & Máximo & \multirow{2}{*}{ p-valor } \\
\hline 0 meses & $98,7^{(\mathrm{a})}$ & 1,86 & 96 & 101 & \\
\hline 3 meses & $97,0^{(a)}$ & 2,1 & 95 & 100 & \multirow{2}{*}{0,5252} \\
\hline 6 meses & $98,3^{(a)}$ & 1.21 & 97 & 100 & \\
\hline 9 meses & $97,5^{(a)}$ & 2,26 & 95 & 101 & \\
\hline 12 meses & $98,3^{(a)}$ & 1,75 & 96 & 101 & \\
\hline
\end{tabular}

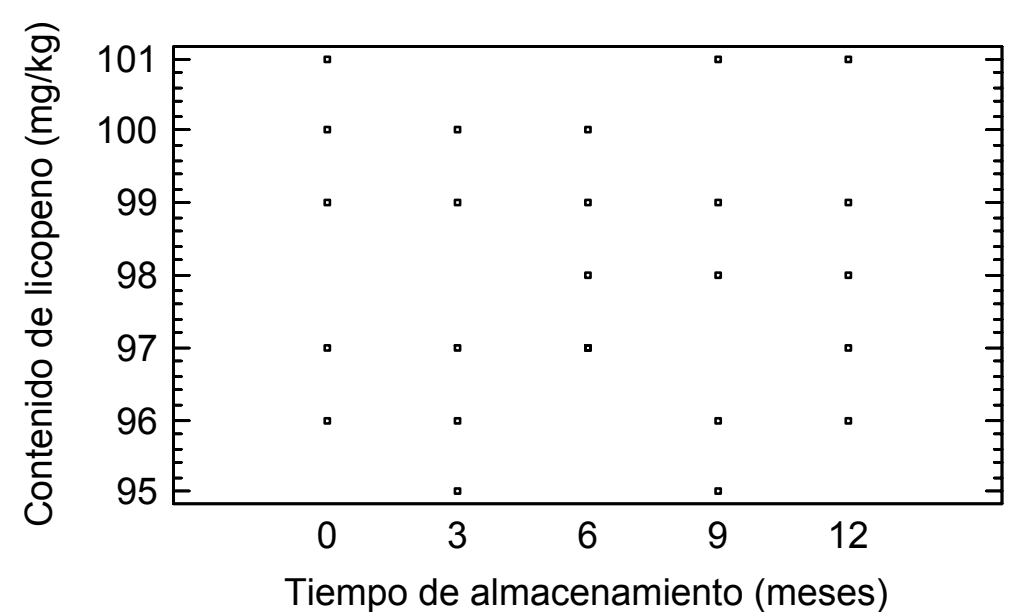

Fig. 3: Gráfico de dispersión de la variación de licopeno a lo largo de su almacenamiento a temperatura ambiente.

El estudio del contenido de licopeno a lo largo del proceso de elaboración de conservas de tomate perita entero pone de manifiesto que el mismo no sufre pérdida por temperaturas elevadas, sino que la misma produce un efecto positivo, en contraposición a lo expuesto por Nguyen y Schwart (1999) y Shi y Le Maguer (2000) acerca de que los factores físicos y químicos afectan la estabilidad de los carotenoides, especialmente temperaturas elevadas, exposición a la luz, al oxígeno y a $\mathrm{pH}$ extremos. La estabilidad observada durante el almacenamiento podría estar relacionada con el tipo de envasado (en hojalata) y el bajo nivel de oxígeno presente debido al proceso de expulsión que se realiza durante la dosificación del líquido de cobertura. 


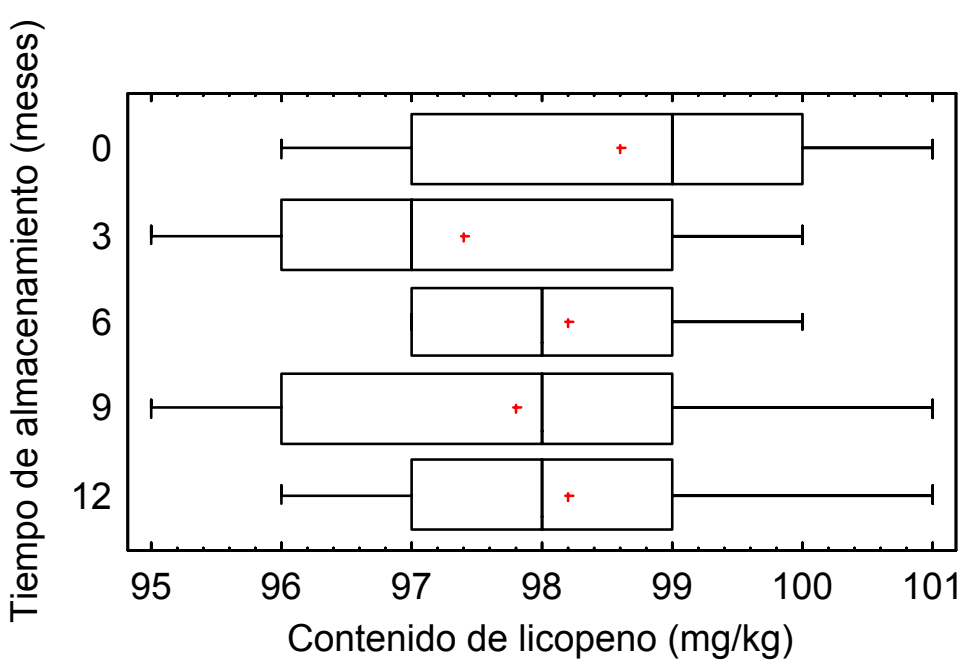

Fig. 4: Gráfico de Cajas y Bigotes de la variación de licopeno a lo largo de su almacenamiento a temperatura ambiente.

\section{CONCLUSIONES}

Durante el proceso de industrialización de tomates peritas pelados en conserva la operación de pelado por escaldado provoca una disminución del contenido de licopeno lo que indica la importante presencia del carotenoide en la epidermis del fruto.

La operación de esterilización industrial en tomates peritas pelados enteros en conserva, produce liberación celular del carotenoide licopeno, manteniendo su estabilidad durante el almacenamiento. Este comportamiento, combinado con el aumento de la biodisponibilidad del licopeno debido a la cocción reportado por otros autores, aporta fundamento científico para considerar a estas conservas alimento funcional natural.

\section{REFERENCIAS}

Candelas, M.G. y otros cuatro autores; Contenido de licopeno en jugo de tomate secado por aspersión, Revista Mexicana de Ingeniería Química: 4, 299-307 (2005).

Cohn, W. y otros cinco autores; Comparative multiple dose Plasma kinetics of lycopene administered in tomato juice, tomato soup or lycopene tablets. Eur. J. Nutr.: 43, 304-312 (2004).

Fornelli, F. y otros cuatro autores; The influence of lycopene on the proliferation of human brast cell line (MVF/), Toxicol in vitro: 21(2), 217-223 (2007)

Gartner, Ch., W. Stahl y H. Sies; Lycopene is more bioavailable from tomato paste than from fresh tomatoes. Am. J. Clin. Nutr.: 66, 116-122 (1997).

Granado, F., B. Olmedilla, I. Blanco y E., Rojas-Hidalgo; Carotenoid composition in raw and cooked Spanish vegetables, J. Agric. Food Chem.: 40, 2135-2140 (1992).

Lambelet, P. y otros cuatro autores; Improving the stability Z-isomers in isomerised tomato extracts, Food Chemistry: 112, 156-161 (2008)

Lee A., D.I. Thurnham y M. Chopra; Comsuption of tomato products with olive oil but not sunflower activity of plasma. Free Radic. Biol. Med.: 29(10), 1051-1055 (2000).

Méndez, R.M. y U.H. Hernández; Contenido de licopeno en productos Mexicanos elaborados con jitomate, IV Congreso Internacional de Ingeniería Bioquímica y XV Congreso Nacional de Ingeniería Bioquímica, Moreli, Michoacán, México (2006). 
Micozzi, M. S. y otros siete autores: Plasma carotenoid response to chronic intake of selected foods and ß-carotene supplements in men, Am. J. Clin. Nutr., 55: 1120-1125 (1992).

Nguyen, M. y S. Schwart; Lycopene: chemical and biological properties, Food Technology: 58(2), 3844 (1999).

Olmedilla B.; Licopeno: Fuentes dietéticas y biodisponibilidad en los humanos, Ibérica Actualidad Tecnológica: 424, 535-540 (1999).

Parker, R.S.; Absorption, metabolism, and transport of carotenoids, FASEB J.: 10, 542-551 (1996).

Perking-Veazie, P., J. Collins, D. Pair y W. Roberts; Lycopene content differs among red-fleshed watermelon cultivars, J. Sci. Food Agric.: 81, 983-087 (2001).

Raffo, A. y otros cuatro autores; Seasonal variations in antioxidant components of cherry tomatoes (Lycopersicon esculetum cv. Naomi F1), Journal of Food Composition and Analysis: 19, 11-19 (2006).

Rao, V., Ed.; Tomatoes, Lycopene and Human Health. Ed. Caledonian Press. ISBN: 0-9553565-0-4, 39-62, Bacelona, España (2006).

Rodriguez-Amaya, D.B.; Carotenoids and Food Preparation: The retention of provitamin a carotenoids in prepared, processed and stored foods, U.S. Agency for International Development (USAID) (1997).

Sadler, G., J. Davis y D. Dezman; Rapid Extraction of Lycopene and Carotene from Reconstituted tomato Paste and Pink Grapefruit Homogenates, J. Food Sci.: 55(5),1460-1 (1990).

Schierle J. y otros seis autores; Content and isomeric ratio of lycopene in food and human blood plasma, Food Chemistry: 59, 459-465 (1997).

Shi J. y M. Le Maguer; Lycopene in Tomatoes: Chemical and Physical properties affected by food processing, Critical Review in Biotechnology: 20 (4), 293-334 (2000).

Stahl, W. y H. Sies; Uptake of lycopene and its geometrical isomers is greater from heat-processed han from unprocessed tomato juice in humans, J. Nutr.: 122, 2161-2166 (1992).

Van het Hof K.H. y otros siete autores; Carotenoid bioavailability in humans from tomatoes processed in different ways determined from the carotenoid response in the triglyceride-rich lipoprotein fraction of plasma after a single consumption and in plasma after four days of consumption, J. Nutr.: 130, 1189-1196 (2000).

Zapata, L.M., L. Gerard, C. Davies y M.C. Schvab; Estudio de los componentes antioxidantes y actividad antioxidante en tomates, Ciencia, Docencia y Tecnología: 35, 173-193 (2007). 
